\title{
Nonlinear Optimal Sliding Mode Midcourse Controller with Thrust Vector Control
}

\author{
Fu-Kuang Yeh',Hsiuan-Hau Chien ${ }^{1}$, and $\mathrm{Li}$-Chen $\mathrm{Fu}^{1,2}$ \\ Department of Electrical Engineering' \\ Department of Computer Science and Information Engineering ${ }^{2}$ \\ National Taiwan University, Taipei, Taiwan, R.O.C. \\ Email: lichen@ccms.ntu.edu.tw
}

\begin{abstract}
In this paper, a nonlinear midcourse missile controller with Thrust Vector Control (TVC) inputs for the interception of a theater ballistic missile is presented. First, an optimal midcourse guidance law is designed to minimize the control effort and the distance between the missile and the target. Then, a quaternion-based sliding-mode attitude controller is proposed to track the attitude command and to cope with the effects from variations of missile's inertia, aerodynamic force, and wind gusts. The exponential stability of the overall system is analyzed thoroughly via Lyapunov stability theory. Extensive simulations are conducted to validate the effectiveness of the proposed guidance law and the associated TVC. Keywords: Sliding Mode, Optimal Control, Target Tracking, Nonlinear System, Quatemion
\end{abstract}

\section{Introduction}

Based on the concept of the PN guidance law, the constant bearing guidance is often employed on the Bank-to-Turn (BTT) missiles [1], whereas a different kind of guidance law, namely, zero-sliding guidance law aims at eliminating the sliding velocity between the missile and the target in the direction normal to LOS [2]. $\mathrm{Ha}$ and Chong derived a new command to line-of-sight (CLOS) guidance law for short-range surface to air missile via feedback linearization [3] and its modified version [4] with improved performance.

In order to utilize the prior information on the future target maneuvers or on the autopilot lags, a new optimal guidance law without estimation of the interception time is proposed to deal with situation where the accurate time-to-go is unavailable [8].Attitude control is another important issue to be addressed for successful missile operation. It is quite often that quatemion representation has been adopted to describe the attitude of a spacecraft [9]. To account for the non-ideal factors of the spacecraft under attitude control and to strengthen the robust property of the controller, the sliding mode control has been employed by Chen and Lo [11], which is then followed by a smooth version [12] incorporating a sliding layer as has been proposed by [6] to avoid the chattering phenomenon, but at the price of slightly degrading the accuracy of the tracking system.

A missile equipped with TVC can effectively control its acceleration direction $[2,14,15]$ when the missile built with fins fails, which in tum implies that the maneuverability and controllability of the missile can be greatly enhanced during the stage when the speed of the missile is slow and/or the air density around the missile is low. Thus, the midcourse guidance employs the TVC is common, but subject to a limitation that the control force is then constrained by the TVC mechanical structure, which further complicates the controller design.

\section{Equations of Motion}

The motion of a missile can be described in two parts as follows:

Translation:

$$
\dot{v}_{M}=a_{M}+g_{M}, \dot{r}_{M}=v_{M}
$$

Rotating:

$$
J \dot{\omega}=-\dot{j} \omega-\omega \times(J \omega)+\bar{T}_{b}+d
$$

where all the variables are defined in Nomenclature.

After referring to Fig. 1, the force and torque exerted on the missile can be expressed in the body coordinate frame, respectively, as

$$
\bar{F}_{b}=N\left[\begin{array}{lll}
\cos d_{p} \cos d_{y} & \cos d_{p} \sin d_{y} & \sin d_{p}
\end{array}\right]^{\gamma}
$$

and

$$
\begin{aligned}
\bar{T}_{b} & =L_{b} \times \bar{F}_{b}+M_{b} \\
& =I N\left[\begin{array}{lll}
M_{h s} / I N & \sin d_{p} & -\cos d_{p} \sin d_{y}
\end{array}\right]^{T}
\end{aligned}
$$

where $M_{4}=\left[\begin{array}{lll}M_{\mathrm{br}} & 0 & 0\end{array}\right]^{T}$ is an external moment command in the axial direction of the missile.

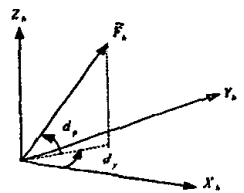

Fig.1. Two Angles of TVC in Body Coordinate

\section{Guidance Law Design}




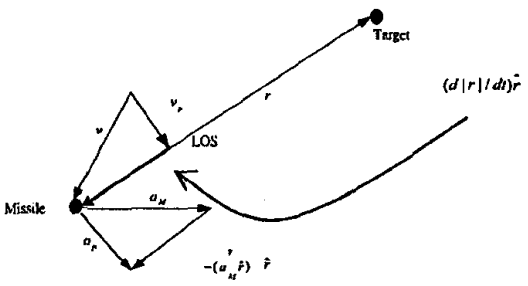

Fig. 2. Relative Acceleration Along the LOS

A modified optimal guidance law without estimation of time-to-go is derived in the following based on the component of the relative velocity normal to the LOS, i.e., $v_{p}=v-\left(v^{r} \hat{r}\right) \hat{r}$. To proceed, we first derive the equation of the relative motion perpendicular to the LOS as follows: (referring to Fig. 2)

$$
\dot{v}_{p}(t)=-a_{p}-\frac{1}{|r|}\left|v_{p}\right|^{2} \hat{r}-\frac{v^{T} \tilde{r}}{|r|} v_{p},
$$

where $a_{p}=a_{M}-\left(a_{M}^{r} \hat{r}\right) \hat{r}$ denotes acceleration of the missile perpendicular to the LOS. Referring to (5), the perpendicular acceleration component $a_{p}$ is specified as

$$
a_{p}=-u-\frac{v^{T} \hat{r}}{|r|} v_{p}
$$

where $u$ is another exogenous design input calculated by minimizing the quadratic cost function defined as

$J=\frac{1}{2} \gamma(T) v_{p}^{T}(T) v_{p}(T)+\frac{1}{2} \int_{0}^{T}\left(\sigma v_{p}^{T}(t) v_{p}(t)+\rho u^{r}(t) u(t)\right) d t(7)$

where $\gamma(T) \geq 0, \sigma>0, \rho>0$, and $\left[t_{0}, T\right]$ is the time interval over which we are interested in the behavior of the missile interception. By optimal control theory [5], the acceleration component perpendicular to the LOS in (6) is

$$
a_{p}(t)=\left[\sqrt{\frac{\sigma}{\rho}\left(1+\frac{2}{e^{\sqrt[3]{\frac{\sigma}{\rho}}(r-1)}-1}\right)}-\frac{v \dot{r}}{\mid r}\right] v_{p}(t)
$$

so that the equation of the relative motion in (5) becomes

$$
\dot{\nu}_{p}=-\sqrt{\frac{\sigma}{\rho}}\left(1+\frac{2}{e^{\sqrt[2]{\frac{\sigma}{\rho}(r-1)}-1}}\right) v_{p}-\frac{1}{|r|}\left|\nu_{p}\right|^{2} \hat{r}
$$

which is well defined except at $r=0$. The resulting dynamics in above will testify that the presented guidance law will ensure target interception under some appropriate condition. This is stated in Lemma 1 as follows.

Lemma 1: Let the equation of the relative motion perpendicular to the LOS and the modified optimal guidance law be given by (5) and (8), respectively. If $v$ has no component on the normal direction of the LOS, and $v^{T} r \leq \beta<0$ with $v$ being bounded away from zero, then the ideal midcourse guidance system will ensure target reaching.

Proof: See Appendix A.

\section{Autopilot System Design}

The dynamic model of a missile, treated as a rigid body, can be derived as

$$
\begin{aligned}
& \dot{\bar{q}}_{e}=\frac{1}{2}\left\langle\bar{q}_{e} \times\right\rangle \omega_{e}+\frac{1}{2} q_{e 4} \omega_{e} \\
& \dot{q}_{e 4}=-\frac{1}{2} \omega_{e}^{T} \bar{q}_{e} \\
& J \dot{\omega}=-j \omega-\omega \times(J \omega)+\bar{T}_{b}+d
\end{aligned}
$$

where $\omega_{e}=\omega-\omega_{d}$ is the error between angular velocities at the present attitude and the desired attitude, respectively.

Step 1: Let us choose the sliding manifold as

$$
S_{a}=P \bar{q}_{e}+\omega_{e}
$$

where $P=\operatorname{diag}\left[\begin{array}{lll}p_{1} & p_{2} & p_{3}\end{array}\right]$ is a positive definite diagonal matrix. If the sliding mode condition is kept, then the system dynamics are then constrained by the following differential equations

$$
\begin{aligned}
& \dot{\bar{q}}_{e}=-\frac{1}{2}\left\langle\bar{q}_{e} \times\right\rangle P \bar{q}_{e}-\frac{1}{2} q_{e 4} P \bar{q}_{e} \\
& \dot{q}_{e 4}=\frac{1}{2} \bar{q}_{e}^{T} P \bar{q}_{e}
\end{aligned}
$$

It has been shown that [2], the system origin $\left(\bar{q}_{e}, w_{e}\right)=\left(0_{3 \times 1}, 0_{3 \times 1}\right)$ of the ideal system (12) is indeed exponentially stable.

Step 2: Assume that $J$ is symmetric and positive definite, and let the candidate of a Lyapunov function be set as

$$
V_{s}=\frac{1}{2} S_{a}^{T} J S_{a},
$$

where $S_{a}=\left[\begin{array}{lll}S_{a 1} & S_{a 2} & S_{a 3}\end{array}\right]^{T}$.

Let the control law be proposed as

$\bar{T}_{b}=-J_{0} P\left(\frac{1}{2}\left\langle\bar{q}_{e} \times\right) \omega_{e}+\frac{1}{2} q_{e t} \omega_{e}\right)+\omega \times\left(J_{0} \omega\right)+J_{0} \dot{\omega}_{d}+\tau$

where $\tau=\left[\begin{array}{lll}\tau_{1} & \tau_{2} & \tau_{3}\end{array}\right]^{T}, \tau_{i}=-k_{i}\left(q, \omega, q_{d}, \dot{q}_{d}, \ddot{q}_{d}\right) \operatorname{sgn}\left(S_{a j}\right)$, $\operatorname{sgn}(D)$ is a sign function, and properly chooses $k_{l}(\cdot)$, $i=1,2,3$, then the time derivative of $V_{x}$ can be evaluated as :

$$
\dot{\nu}_{s}=S_{a}^{T}[\delta+\tau] \leq-\sum_{i=1}^{3}\left|S_{a i}\right|\left[k_{i}-\delta_{i}^{\max }\right]<0
$$

where 


$$
\begin{aligned}
\delta= & -\dot{J}_{\omega}-\omega \times(\Delta \omega)+d-\Delta \dot{\omega}_{d}+\frac{1}{2} j s_{a} \\
& +\Delta J P\left(\frac{1}{2}\left(\bar{q}_{e} \times\right\rangle \omega_{e}+\frac{1}{2} q_{e 4} \omega_{e}\right)
\end{aligned}
$$

and $k_{1}(\cdot), i=1,2,3$, are chosen sufficiently large as will be commented below. Let the external disturbances $d$ and the magnitudes of $j$ and $\Delta J$ be all bounded, then the bounding function on $\left|\delta_{i}\right|$, which obviously is a function of $q, \omega, q_{d}, \dot{q}_{d}$ and $\ddot{q}_{d}$, can be found and represented as $\delta_{i}^{\max }\left(q, \omega, q_{d}, \dot{q}_{d}, \ddot{q}_{d}\right) \geq\left|\delta_{i}\right|$. It is evident that if we choose $k_{i}\left(q, \omega, q_{d}, \dot{q}_{d}, \ddot{q}_{d}\right)>\delta_{i}^{\max }\left(q, \omega, q_{d}, \dot{q}_{d}, \ddot{q}_{d}\right)$ for $i=1,2,3$, the reaching and sliding conditions of the sliding mode $S_{a}=0$ are guaranteed.

In other words, if the desired acceleration is $a_{p}+\bar{r}$, and here we assume that the flying direction will be in alignment with the axial direction of the missile, then the relationship between the actual acceleration $a_{\mu}$ applied on the missile and the desired acceleration $a_{p}+\bar{r}$ is the following:

$$
a_{M}=B_{b}(q) B^{T}\left(q_{E}\right) B_{b}^{T}(q)\left(a_{p}+\bar{r}\right)
$$

Remark: Recall that $B_{b}(q)$ is the transformation from the current body coordinate to the inertial coordinate, and $B\left(q_{e}\right)$ is the transformation from the current body coordinate to the desired body coordinate.Since the actual acceleration exerted on the missile is $a_{M}$, the component of the actual acceleration perpendicular to the LOS is

$$
a_{M p}=\frac{\left\{\left[B_{b}(q) B^{T}\left(q_{e}\right) B_{b}^{T}(q)\left(a_{p}+\bar{r}\right)\right]^{T} a_{p}\right\} a_{p}}{\left|a_{p}\right|^{2}},
$$

where $a_{p}$, in (8), is the desired acceleration perpendicular to the LOS as has been mentioned before. As a result, we get a new state equation from (5) as follows:

$$
\dot{v}_{p}=\frac{\left\{\left[B_{b}(q) B^{T}\left(q_{e}\right) B_{b}^{T}(q)\left(a_{p}+\tilde{r}\right)\right]^{T} a_{p}\right\} a_{p}}{\left|a_{p}\right|^{2}}-\frac{1}{|\gamma|}\left|v_{p}\right|^{2} \dot{r}-\frac{v^{T} \hat{r}_{\hat{r}}}{|\gamma|}(
$$

To evaluate the stability of the relative velocity component $\nu_{p}$, we define a Lyapunove function $V_{G}$ as

$$
V_{G}=\frac{1}{2} v_{p}{ }^{T} v_{p} .
$$

Then, the time derivative of the Lyapunov function can be derived as

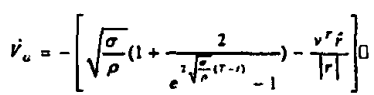

$$
\begin{aligned}
& v_{p}^{T} v_{p}\left[l+\frac{2\left[\bar{B}^{T}\left(q_{c}\right) F_{b}\right]^{T} \bar{a}_{p}}{\left|a_{p}\right|^{2}}\right]-\frac{v^{T} \hat{r}^{2}}{|\gamma|} v_{p}^{T} v_{p}
\end{aligned}
$$

where $a_{p}$ is given as in (8), $F_{b}=B_{d}{ }^{\top}\left(a_{p}+\bar{r}\right)$, $B_{b}^{T}=B_{b}^{-1}, \bar{a}_{p}=B_{1}^{r} a_{\rho}, \bar{B}\left(q_{e}\right)=\left\langle\bar{q}_{0} \times\right\rangle\left\langle\bar{q}_{0} x\right\rangle$. Note that we use the fact that $v_{p}^{r} \hat{r}=\bar{r}^{r} a_{p}=0$, and $B\left(q_{e}\right)=I_{3 \times 3}+2\left(\bar{q}_{e} \times\right\rangle\left\langle\bar{q}_{e} \times\right)+2 q_{e 4}\left\langle\bar{q}_{e} \times\right\rangle$.

To verify the stability of the overall system, we define a Lyapunov function candidate of the overall system as the sum of the previously defined functions $v$, and $v_{G}$, i.e.,

$$
v=V_{s}+V_{G},
$$

The time derivative of the Lyapunov function can be derived as

$$
\begin{aligned}
& \dot{V}=S_{a}^{J}\left[-j \omega-\omega \times(J a)+\bar{T}_{b}+d-J \dot{a}_{d}+J P\left(\frac{1}{2}\left\langle\bar{g}_{e} x\right) \alpha_{e}+\frac{1}{2} q_{e 4} a_{e}\right)\right. \\
& \left.+\frac{1}{2} \dot{S}_{l}\right]-K_{1}\left(v_{p}, T, t\right)-K_{2}\left(v_{p}, v_{,}, T, T, t\right) \frac{\left.2 \bar{B}^{T}\left(q_{e}\right) F_{b}\right|^{T} \bar{a}_{p}}{\left|a_{p}\right|^{2}}
\end{aligned}
$$

referring to Eqs. (15) and (19), where

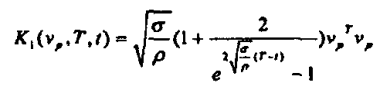

$$
\begin{aligned}
& =\bar{K}_{1}(T, t) v_{p}^{T} \nu_{p}>\sqrt{\frac{\sigma}{\rho}} \nu_{p}{ }^{T} v_{p} \geq 0 \\
& K_{2}\left(v_{p}, v, r, T, t\right)=\left[\sqrt{\frac{\sigma}{\rho}}\left(3+\frac{2}{e^{\left.\sqrt[3]{\frac{\sigma}{\rho}}, r-1\right)}-1}-\frac{v^{r} \hat{r}}{|r|}\right] v_{p} v_{p}\right. \\
& =\bar{K}_{2}(v, r, T, t) v_{p}^{T} \nu_{p}>\sqrt{\frac{\sigma}{\rho}} \nu_{\rho}^{T} \nu_{p} \geq 0
\end{aligned}
$$

so that

$$
\begin{aligned}
& \dot{V}=S_{a}\left[-j \omega-\omega \times(J a)+\bar{T}_{b}+d-J \dot{\omega}_{b}+J \frac{p}{2}\left(\left(\bar{q}_{c}\right\rangle \alpha_{l}+q_{t} \omega_{2}\right)\right.
\end{aligned}
$$

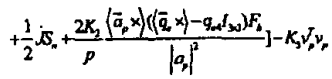

where $K_{3}=K_{3}\left(v, r, q_{e}, w_{e}, a_{p}, T, l\right)$ is defined as :

$K_{3}=\bar{K}_{1}+\frac{2 \bar{K}_{2}}{p} \frac{F_{h}^{T}\left(\left\langle\bar{q}_{c} x\right)+q_{e t} I_{3 \times 3}\right)\left(\bar{a}_{p} x\right\rangle}{\left|a_{p}\right|^{2}} \omega$, and the matrix $P$ is chosen to be $P=p \times I_{3 \times 1}$.

Theorem 1: Let the modified optimal guidance law be proposed as in (8), (18) and the torque input of the autopilot is given as follows:

$\bar{T}_{b}=-J_{0} p\left[\frac{1}{2}\left\langle\bar{q}_{e} \times\right\rangle \omega_{e}+\frac{1}{2} q_{e+} \omega_{c}\right]+\omega \times\left(J_{0} \omega\right)+J_{0} \dot{\omega}_{d}+\tau^{\prime}(24)$ 
where $\tau^{\prime}=\left[\begin{array}{lll}\dot{\tau}_{1} & i_{2}^{\prime} & \tau_{3}^{\prime}\end{array}\right]^{\tau}, \quad \dot{\tau}_{i}^{\prime}=-\bar{k}_{t}\left(q, \omega, q_{d}, \dot{q}_{d}, \ddot{q}_{d}, v_{p}, v, r, T, t\right)$ $\operatorname{sgn}\left(S_{a r}\right)$ for some appropriate functional gains $\bar{k}_{i}, i=1,2,3$, and $p$ is chosen to be large enough. If $v$ is such that $v^{T}\left(t_{0}\right) \hat{r}\left(t_{0}\right)<0$, where $t_{0}$ is the starting time and $v$ is bounded away from zero, then the integrated overall midcourse guidance and autopilot system will be stable and the target reaching property is achieved.

Proof: After substitution of the torque input (24) by hypothesis, the expression of $\dot{V}$ can be readily simplified as:

$$
\dot{V}=S_{a}^{T}\left[\bar{\delta}+\tau^{\prime}\right]-K_{3} v_{p}^{r} v_{p}
$$

where

$$
\begin{gathered}
\bar{\delta}=-j \omega-\omega \times(\Delta J \omega)+d-\Delta J \dot{\omega}_{d}+p \Delta J\left(\frac{1}{2}\left\langle\bar{q}_{e} \times\right\rangle \omega_{e}\right. \\
\left.+\frac{1}{2} q_{s 4} \omega_{e}\right)+\frac{1}{2} j S_{a}+\frac{2 K_{2}}{p} \frac{\left\langle\bar{a}_{p} \times\right\rangle\left\langle\left(\bar{q}_{s} \times\right\rangle-q_{e d} l_{3 \alpha 3}\right) F_{b}}{\left|a_{p}\right|^{2}}
\end{gathered}
$$

Since the external disturbance $d$ and the uncertainties $j$ and $\Delta J$ are all bounded by assumption, we conclude that the upper bounds $\bar{\delta}_{1}^{\max }$, $\bar{\delta}_{i}^{\max } \geq\left|\bar{\delta}_{i}\right|, i=1,2,3$, are functions of $q, \omega, q_{d}, \dot{q}_{d}$, $\ddot{q}_{d}, v_{p}, v, r, T$ and $t$. It is evident that if the functional gain $\bar{k}_{i}\left(q, \omega, q_{d}, \dot{q}_{d}, \ddot{q}_{s}, v_{p}, v, r, T, t\right)>\max \left\{\delta_{i}^{\max }\right.$, $\left.\bar{\delta}_{i}^{\text {mux }}\right\}+\eta_{i} i=1,2,3$, for $\eta_{i}>0$, referring to Eqs. (16) and (26), can be chosen, then (15) apparently holds and the expression (25) can be further explored as:

$$
\dot{V}=-\sum_{i=1}^{3} S_{a \mid} \mid\left\{\bar{k}_{i}-\delta_{i} \operatorname{sgn}\left(S_{a x}\right)\right]-K_{3} v_{p}^{T} v_{p}
$$

Working Lemma 2: Through the entire midcourse phase, if $v^{T} \hat{r}<0$, with $v$ being bounded away from zero, then we can always find appropriate gain $p$ and the adjustable convergent time parameter $T>t$ such that

$$
K_{3}=\bar{K}_{1}+\frac{2 \bar{K}_{2}}{p} \frac{F_{h}^{r}\left(\left(\bar{q}_{\mathrm{t}} x\right)+q_{\mathrm{es}} l_{3 \mathrm{~s}}\right)\left\langle\bar{a}_{p} x\right\rangle}{\left|a_{\mathrm{p}}\right|^{2}} \omega_{\mathrm{e}} \geq K_{30}>0
$$

for all $r \geq 0$.

Proof: See Appendix A.

As a result, (28) can be expressed as :

$$
\dot{v} \leq-\sum_{i=1}^{3} \eta_{i}\left|S_{a 1}\right|-K_{30} v_{p}^{r} v_{p}
$$

which means that $-\dot{V}$ is positive definite, and hence $s_{a} \rightarrow 0, v_{p} \rightarrow 0$ as $t \rightarrow \infty$ via use of Lyapunov stability theory.

Finally, to show that Lemma 1 is satisfied, i.e., target reaching, we need to show that $\nu_{\hat{r}} \leq \beta<0$ at all time. First, $v_{p}$ has to be verified to be exponentially decaying. Although $\dot{v}$ and $\dot{v}_{s}$ can be shown negative definite via Theorem 1 via torque input (24), by definition $\dot{V}=\dot{V}_{G}+\dot{V}_{S}$ in (21) $\dot{V}_{G}$ can not be proved negative definite directly. To establish such a fact, we derive $\dot{V}_{G}$ explicitly from Eqs. (19), and (21) (23), i.e.,
$\dot{V}_{G}=-\left[\bar{K}_{l}-\frac{2 \bar{K}_{2}}{p} \frac{F_{b}^{T}\left(\left\langle\bar{q}_{e} \times\right)+q_{e 4} I_{3 \times 3}\right)\left\langle\bar{a}_{p} \times\right\rangle}{\left|a_{p}\right|^{2}}\left(S_{a}-\omega_{e}\right)\right] v_{p}^{T_{p}}(2$

By use of the proof Lemma 2, the maximum value $\bar{P}_{\max }$ in (29) can also be obtained as

$$
\bar{P}_{\max } \geqslant\left|\frac{F_{b}^{T}\left(\left(\bar{q}_{e} \times\right)+q_{e 4}(3 \times 3)\left(\bar{a}_{p} \times\right)\left(s_{a}-a_{e}\right)\right.}{\left|a_{p}\right|^{2}}\right|
$$

where we already showed that $s_{a} \rightarrow 0$ and $\omega_{e} \rightarrow 0$ as $t \rightarrow \infty$ due to the autopilot system design in section 4 referring to (11) and (15). Thus, if the inequality (A4) in Lemma 2 can be modified as

$$
P>2 \max \left\{P_{\max }, \bar{p}_{\max }\right\} \cdot \bar{K}^{\max }
$$

then $\dot{V}_{G}, \dot{V}$, and $\dot{V}_{s}$ are all negative definite, which means $v_{p}$ will be attenuated exponentially at all time due to the definition of

$$
v_{G}=\frac{1}{2} v_{p}^{T} v_{p}
$$

which is always positive definite.

By defining the angle, $\theta$, between $\nu$ and $v_{r}$ referring to Fig. 5 as

$$
\theta=\tan ^{-1}\left(\frac{v_{p} \mid}{\left|p_{r}\right|}\right),
$$

thus we can conclude that $\left|v_{p}\right|$ will decay exponentially referring to $(29)-(31)$, and $\left|v_{r}\right|=\left|v-v_{p}\right| \geq|v|-\left|v_{\rho}\right|$ due to $v=v_{p}+v_{r}$ referring to Fig. 3. Therefore, $\left|\nu_{r}\right|$ will be an increasing function with respect to time, which implies, in tum, the angle $\theta$ will be monotonically decreasing as time goes on. Hence, we can conclude that $\nu^{T} \hat{r}<0$ for all $t \geq 0$ since $v^{T}\left(t_{0}\right) \hat{r}\left(t_{0}\right)<0$ which justifies our assumption in Lemma 1. Therefore, the target tracking objective can be achieved as claimed by the aforementioned theorem.

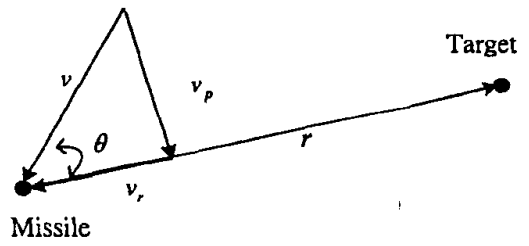

Fig. 3. Relative Velocity between the Target and

the Missile

\section{Simulations}

Q.E.D.

We assume the target is launched from somewhere $600 \mathrm{~km}$ far away from the missile base. The missile has a sampling period of $10 \mathrm{~ms}$. The bandwidth of the TVC is $5 \mathrm{~Hz}$ and the two angular displacements are both limited to $5^{\circ}$. Here, we 
consider the variation of the moment of inertia of the missile. Thus, the inertia matrix including the nominal part $J_{0}$ and the uncertain part $\Delta J$ used here is as follows:

$$
J=J_{0}+\Delta J\left(\mathrm{~kg} \cdot \mathrm{m}^{2}\right)
$$

where

$J_{0}=\left[\begin{array}{ccc}1000 & 100 & 200 \\ 100 & 2000 & 200 \\ 200 & 200 & 2000\end{array}\right], \quad \Delta=\left[\begin{array}{ccc}100 & 100 & 200 \\ 100 & 200 & 200 \\ 200 & 200 & 200\end{array}\right]$

and the variation of the inertial matrix is as

$$
j=\left[\begin{array}{lll}
-1 & -1 & -2 \\
-1 & -2 & -2 \\
-2 & -2 & -2
\end{array}\right]
$$

The initial conditions are set as $q=\left[\begin{array}{llll}0 & -0.707 & 0 & 0.707\end{array}\right]^{T}, \omega(0)=\left[\begin{array}{lll}0 & 0 & 0\end{array}\right]^{T}$, and the variation of missile's mass is as $\dot{m}=-4(\mathrm{~kg} / \mathrm{sec})$ for the initial mass $m=600(\mathrm{~kg})$. Further, we also consider the aerodynamic force and wind gusts exerted on the missile by $d_{1}(t)=\sin (t)+10(u(t-20)-u$ $(t-21))(\mathrm{Nt}-\mathrm{m})$ for $i=1,2,3$, where $u(t)$ is the step function. Our objective here is to drive the missile close to the target within the range of $9 \mathrm{~km}$. The simulation time is 93.13 sec.. The feasibility of the presented approach is satisfactorily demonstrated by the simulation results in Fig. 4 .
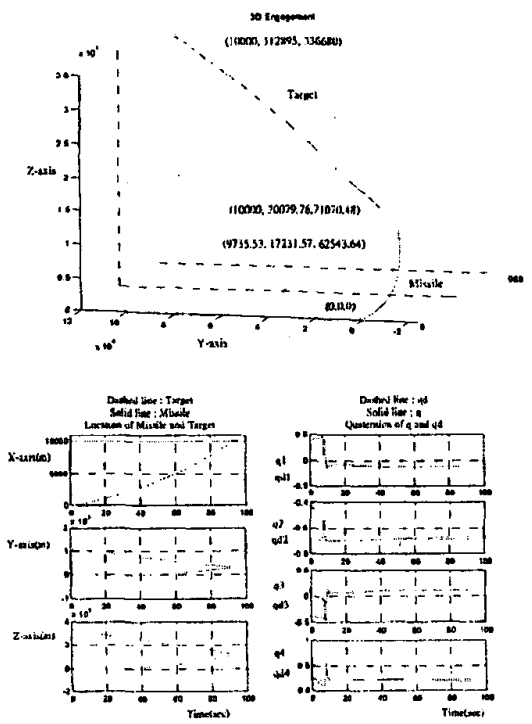

Fig. 4. Simulation Results

\section{Conclusions}

In this paper, we focus on the midcourse phase of the interception, which is a period of time lasting until the missile is close enough to the target such that the sensor located on the missile can lock on the target.
We prove the stability of the individual guidance system, the autopilot system, and the overall system, respectively, via Lyapunov stability theory. Computer simulations have been conducted to verify the feasibility of the integrated midcourse guidance and control system with TVC.

\section{Reference}

[1]Guidance and Adaptive Autopilot Design for BTT Missiles", Proc. Amercion Control Conference, pp.27742778, 1997.

[2]Li-Chen Fu, Chi-Wang Tsai and Fu-Kuang Yeh, "A Nonlinear Missile Guidance Controller with Pulse Type Inpur Devices", Proc. Amercion Contol Conference, pp.3753-3757, 1999.

[3] Ha, L, and Chong $S$, 'Design of a CLOS Guidance Law via $F$ Li-Chen Fu and Wei-Der Chang "A Nonlinear Constant Bearing ooclback Linearization", IEEE Thans on Aeraspace and Electronic Systems, vol 28, na. 1,pp. 51-63, 1992.

[4]Jie Huang and Ching-Fang Lin, "A Modified CLOS Guidance Law Via Right Inversion", IEEE Thans. on Aeraspace and Electronic Sjstems, vol. 31, no. 1, pp. 491-495, 1995.

[5]Frank L. Lewis and Vassilis L. Symos, Optimal Control, John Wiley \& Sons, Inc., 1995.

[6]Slotine, JJE, "Sliding Controller Design for Nonlinear Systems", Int J. Contro, vol 40, no. 2, pp.421-434, 1984.

[7]I. Rusrak and L Meir, "Optimal Guidance for Acoeleration Constrained Missile and Manearvering Target", IEEE Trans. on Aeraspace and Electronic Systems, vol. 26, no. 4, pp.618624, 1990.

[8]Ki Baek Kim, Myung-Joon Kim and Wook Hyun Kwom, "Modern Guidance Laws via Receding Horizon Control without the time-to-go", Proc the $37^{\text {th }}$ IEEE Corff. on Decision \& Control, Tampa, Florida, USA, pp. 4202-4207, 1989.

[9]B. Wie, H. Weiss and A. Arapostathis, "Quaternion Feerlback Regulator for Spacecraft Eigenaxis Rotations", Joumal of Gridance, vol 12, no. 3,pp. 375-380, 1989.

[10]Kuang-Yow Lian, Li-Sheng Wang and Li-Chen Fu, "Globally Valid Adaptive Controllers of Mechanical Systems", IEEE Thans of Automatic Control, vol 42, no. 8,pp. 1149-1154, 1997.

[11]Yon-Ping Chen and Shih-Che Lo, "Sliding-Mode Controller Design for Spacecraft Atritude Tracking Maneuvers", IEEE Thans. on Aeraspace and Electronic Systems, vol. 29, no. 4, pp.1328-1333, 1993.

[12]Shith-Che Lo and Yon-Ping Chen, "Smooth Sliding-Mode Control for Spacecraft Attitude Tracking Manawers", Joumal of Guidance Control, and Dynamics, vol. 18, no. 6, pp. 1345-1349, 1995.

[13]J. -J. E. Slotine and M. D. Di Benedetro, "Hamitronian Adaptive Control of Spacecraff', IEEE Trans on Aztomatic Conorl, vol. 35, mo. 7,pp 848-852, 1990.

[14]Kevin A. Wise and David J. Broy, "Agile Missile Dynamies and Control", Joumal of Gridance, Control, and Dynamics, val 21, no. 3, pp.441-449, 1998.

[15]Der-Ren Taur and Jeng-Shing Chem, "An Optimal Composite Guidance Strategy for Dogfight Air-to-Air IR Missiles", ALAA Gridance, Navigation and Control Conference and Exhibit, wol I,pp. 662-671,1999.

[16]Arkadi Lichtsinder, Eliezer Kreindlex, and Benjamin Gal-Or, 
'Minimum-Time Maneuvers of Thrust-Vectored Aircraft", Joumal of Guidance, Control, and Dynamics, vol. 21, no. 2, pp. 244-250,1998.

[17]David B. Spencer, "Designing Contimuous-Thust Low-Earth-Ohbit to Geosynchronous-Earth-Oibit transfers", Joumal of Spacecraft and Rockets, vol. 32, no. 6, pp. 1033-1038,1995.

\section{Appendix A}

Proof of Lemma 1: Taking

$$
v_{G}=\frac{1}{2} v_{p}{ }^{T} v_{p}
$$

as a Lyapunov function candidate, it can be easily seen that

$$
\frac{1}{4} v_{p}{ }^{T} v_{p} \leq V_{G} \leq v_{p}{ }^{r} v_{p}
$$

Hence, $V_{G}$ is positive definite, decresent, and radially unbounded. The time derivative of $V_{G}$ along the trajectories of the system is given by

$\dot{v}_{G}=-v_{p}^{r}\left[\sqrt{\frac{\sigma}{\rho}}\left(1+\frac{2}{e^{2 \sqrt{\frac{\sigma}{\rho}}(r-1)}-1}\right)\right] v_{p}-\frac{1}{|r|}\left|v_{p}\right|^{2} v_{p}^{r} \tilde{r} \leq-\sqrt{\frac{\sigma}{\rho}} v_{p}{ }^{r} v_{p}$

where $v_{p} r=0$ and $\sqrt{\frac{\sigma}{\rho}}$ is a positive constant.

Thus, $\dot{V}_{G}$ is apparently negative definite, and hence via Lyapunov stability theory, we can conclude that the origin of $v_{p}$ is globally exponentially stable.

$$
\text { We take } V_{r}=\frac{1}{2} r^{T} r \text { as another Lyapunov function }
$$

candidate. Thus, it can be easily seen that $V_{r}$ is positive definite, decrescent, and radially unbounded, then the time derivative is as follows:

$$
\dot{V}_{r}=v_{r}^{T} r=\left(\nu-v_{p}\right)^{T} r=v^{T} r<0
$$

where $v_{r}=\nu-v_{p}$, so that $\dot{v}_{r}$ is negative definite. Therefore, via the Lyapunov stability theory and constant bearing condition [2], the ideal midcourse guidance will render the origin of the missile interceptions system globally exponentially stable.

Proof of Lemma 2 : Since $\frac{a_{p}}{\left|a_{p}\right|}, q_{\alpha}$, and $\omega_{e}$ are bounded,

and $\omega_{e} \rightarrow 0$ as $t \rightarrow \infty$, we have both $\frac{F_{b}^{T}}{\left|a_{p}\right|}=\frac{\left[B_{b}^{T}\left(a_{p}+\bar{r}\right)\right]^{T}}{\left|a_{p}\right|}$ and $\frac{\left\langle\bar{a}_{p} \times\right\rangle}{\left|a_{p}\right|}=\frac{\left\langle\left(B_{b}^{T} a_{p}\right) \times\right\rangle}{\left|a_{p}\right|}$ ane bounded, so that the value of $\frac{F_{b}^{r}\left(\left\langle\bar{q}_{e} x\right\rangle+q_{e d} I_{1 \times 3}\right)\left\langle\bar{a}_{p} x\right\rangle \omega_{e}}{\left|a_{p}\right|^{2}}$ can be concluded to be bounded and converge to zero as $t \rightarrow \infty$. Therefore, the maximum value $p_{\text {max }}$ can be obtained as

$$
p_{\max } \geq\left|\frac{F_{b}^{T}\left(\left\langle\bar{q}_{e} \times\right\rangle+q_{e d} I_{3 x+}\right)\left\langle\bar{a}_{p} \times\right) \omega_{e}}{\left|a_{p}\right|^{2}}\right|
$$

Moreover, $\bar{K}_{1}$ and $\bar{K}_{2}$ are both positive, and

$K_{3}=\bar{K}_{1}\left(1+\frac{1}{p} \frac{2 \bar{K}_{2}}{\bar{K}_{1}} \frac{F_{b}^{T}\left(\left(\bar{q}_{e} \times\right)+q_{e 4} J_{3 \times 3}\right)\left\langle\bar{a}_{p} \times\right\rangle \omega_{e}}{\left|a_{p}\right|^{2}}\right)$ due to the fact of

(22). Hence, the ratio of $\bar{K}_{2}$ to $\bar{K}_{1}$ can be expressed as

$$
\bar{K}=\frac{\bar{K}_{2}}{\bar{K}_{1}}=\frac{\sqrt{\frac{\sigma}{\rho}}\left(1+\frac{2}{e^{2 \sqrt{\frac{\sigma}{\rho}}(r-1)}-1}\right)-\frac{v^{T} \dot{r}}{|r|}}{\sqrt{\frac{\sigma}{\rho}}\left(1+\frac{2}{e^{2 \sqrt{\frac{\sigma}{a}(r-t)}}-1}\right)}
$$

where the assumptions that $v^{r} \hat{r}<0$ and $-\frac{v^{T} \hat{r}}{|r|}=-\frac{1}{|r|} \frac{d}{d t}|r|=-\frac{d}{d t} \ln |r|$ is boundod for $|r| \neq 0$ are satisfied. Therefore, the maximum $\vec{K}^{\text {max }}$ of $\bar{K}$ in (A2) can be found and is denoted as $\bar{K} \leq \bar{K}^{\text {max }}$. Then, (28) can be re-expressed as

$$
\begin{aligned}
& \bar{K}_{3}=\bar{K}_{1}+\frac{2 \bar{K}_{2}}{p} \frac{F_{b}^{r}\left(\left\langle\bar{q}_{e} x\right\rangle+q_{e d} I_{3 \times 0}\right)\left\langle\bar{a}_{p} x\right\rangle}{\left|a_{p}\right|^{2}} \omega_{e}=\bar{K}_{1}(1 \\
& \left.+\frac{2 \bar{K}}{p} \frac{F_{b}^{T}\left(\left\langle\bar{q}_{e} \times\right)+q_{e 4} I_{1 \times 3}\right)\left\langle\bar{a}_{p} x\right\rangle}{\left|a_{p}\right|^{2}} \omega_{e}\right) \geq \bar{K}_{1}\left(1-\frac{2 \bar{K}^{\max }}{p} p_{\max }\right) \\
& \text { Now, if we let } \\
& p>2 p_{\max } \cdot \bar{K}^{\max },
\end{aligned}
$$

which together with that fact that $\bar{K}_{1}$ is lower bounded by a positive constant immediately implies the inequality conclusion (28).

\section{Nomenclature}

\section{a Acoeleration vectox \\ $d$ Distutiancesvector \\ $d_{p}$ Pith angeof propellant \\ $d_{y}$ Yaw angle of propelant \\ $\bar{F}$ Thust vector}

$g$ Gravitational acceleration vector

$J$ Momert of inertial matrix

$J_{0}$ Nominal parts of $J$

$\Delta V$ Varizion of $J$

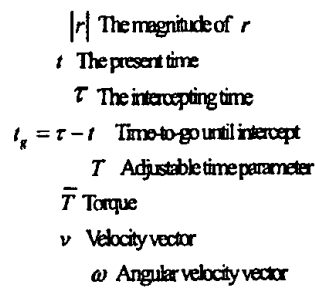
Subscripts

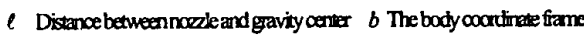
$L_{b}=\left[\begin{array}{lll}-\ell & 0 & 0\end{array}\right]^{\top}$ Disphacemert vector $d$ Desired $m$ Mass of themissile $e$ Eror $N$ Magnitude of thust $i$ The inertial cocntinete frame $q$ Quatemion $M$ Missle

$r$ Position vector frommissileto taget $\quad p$ Papenfoularto $L O S$

$\hat{r}$ The unit vectrof of $r \quad T$ Targa 